\title{
A Characterization of Hilbert SPACES AND the VECTOR-VALUED LITTLEWOOD-PALEY THEOREM
}

\author{
Y.-S. Han and Y. Meyer
}

\begin{abstract}
In this note we prove that the existence of the Banach space-valued Littlewood-Paley theorem implies that a Banach space is isomorphic to a Hilbert space.
\end{abstract}

\section{Introduction}

Suppose that a function $\psi$ is in $S\left(R^{n}\right)$ with supp $\widehat{\psi} \subset\left\{\xi \in R^{n}: \frac{1}{2} \leq|\xi| \leq 2\right\}$ and $|\widehat{\psi}(\xi)| \geq c>0$ if $\frac{3}{5} \leq|\xi| \leq \frac{5}{3}$. Then one form of the classical Littlewood-Paley theorem on $R^{n}$ says

$$
c\|f\|_{p} \leq\left\|\left\{\sum_{k \in Z}\left|\psi_{k} * f\right|^{2}\right\}^{\frac{1}{2}}\right\|_{p} \leq C\|f\|_{p}
$$

where $1<p<\infty, \psi_{k}(x)=2^{k n} \psi\left(2^{k} x\right)$, and $c, C$ are constants independent of $f$.

In this note, we study the vector-valued Littlewood-Paley theorem. To be precise, let $B$ be a Banach space and $L_{B}^{p}\left(R^{n}\right)$ be the space of strongly measurable B-valued functions $f$ for which $|f|_{B} \in L^{p}\left(R^{n}\right)$. It is well-known that if $B$ is a Hilbert space, then the classical Littlewood-Paley theorem still holds:

$$
c\|f\|_{L_{B}^{p}} \leq\left\|\left\{\sum_{k \in Z}\left|\psi_{k} * f\right|^{2}\right\}^{\frac{1}{2}}\right\|_{L_{B}^{p}} \leq C\|f\|_{L_{B}^{p}}
$$

where $1<p<\infty$ and $\psi$ is the same function as in (1.1).

We first prove that if $B$ is a Banach space and (1.2) holds for one function $\psi$ mentioned above, then (1.2) holds for a more general family of operators. More precisely, we need the following definition.

Definition 1.1. A family of operators $\left\{S_{k}\right\}_{k \in Z}$ is said to be an approximation to the identity if for $0<\epsilon \leq 1$ and $\delta=\epsilon-\epsilon^{\prime}>0$ there is a constant $C$ such that for all $k \in Z$ and all $x, x^{\prime}, y$, and $y^{\prime} \in R^{n}, S_{k}(x, y)$, the kernels of $S_{k}$, satisfy the following conditions:

$$
\begin{gathered}
\left|S_{k}(x, y)\right| \leq C \frac{2^{-k \epsilon}}{\left(2^{-k}+|x-y|\right)^{n+\epsilon}} \\
\left|S_{k}(x, y)-S_{k}\left(x^{\prime}, y\right)\right| \leq C\left(\frac{\left|x-x^{\prime}\right|}{2^{-k}+|x-y|}\right)^{\epsilon} \frac{2^{-k \epsilon}}{\left(2^{-k}+|x-y|\right)^{n+\epsilon}}
\end{gathered}
$$

Received April 20, 1995, revised January 24, 1996.

1991 Mathematics Subject Classification: 42B20, 42B25, 46B20, 46C19.

Key words and phrases: Littlewood-Paley theorem, Calderón-type reproducing formula, Calderón-Zygmund theory. 
for $\left|x-x^{\prime}\right| \leq \frac{1}{2}\left(2^{-k}+|x-y|\right)$,

$$
\left|S_{k}(x, y)-S_{k}\left(x, y^{\prime}\right)\right| \leq C\left(\frac{\left|y-y^{\prime}\right|}{2^{-k}+|x-y|}\right)^{\epsilon} \frac{2^{-k \epsilon}}{\left(2^{-k}+|x-y|\right)^{n+\epsilon}}
$$

for $\left|y-y^{\prime}\right| \leq \frac{1}{2}\left(2^{-k}+|x-y|\right)$,

$$
\begin{aligned}
& \left|\left[S_{k}(x, y)-S_{k}\left(x, y^{\prime}\right)\right]-\left[S_{k}\left(x^{\prime}, y\right)-S_{k}\left(x^{\prime}, y^{\prime}\right)\right]\right| \\
& \quad \leq C\left(\frac{\left|x-x^{\prime}\right|}{2^{-k}+|x-y|}\right)^{\epsilon^{\prime}}\left(\frac{\left|y-y^{\prime}\right|}{2^{-k}+|x-y|}\right)^{\epsilon^{\prime}} \frac{2^{-k \delta}}{\left(2^{-k}+|x-y|\right)^{n+\delta}}
\end{aligned}
$$

for $\left|x-x^{\prime}\right| \leq \frac{1}{2}\left(2^{-k}+|x-y|\right)$ and $\left|y-y^{\prime}\right| \leq \frac{1}{2}\left(2^{-k}+|x-y|\right)$, and $\delta=\epsilon-\epsilon^{\prime}>0$,

$$
\int S_{k}(x, y) d y=\int S_{k}(x, y) d x=1 \quad \text { for all } k \in Z \text {. }
$$

All of the conditions (i) $-(\mathrm{v})$ on the approximate identities are needed for the Calderón reproducing formula, namely Theorem 2.1. See [1] for the details.

In this note, we prove

Theorem 1.1. Suppose that $B$ is a Banach space. Suppose $\left\{S_{k}\right\}$ is an approximation to the identity and $D_{k}=S_{k}-S_{k-1}$, and the Littlewood-Paley theorem holds for $\left\{D_{k}\right\}$, that is, for $1<p<\infty$,

$$
c\|f\|_{L_{B}^{p}} \leq\left\|\left\{\sum_{k \in Z}\left|D_{k}(f)\right|^{2}\right\}^{\frac{1}{2}}\right\|_{L_{B}^{p}} \leq C\|f\|_{L_{B}^{p}} .
$$

Then the Littlewood-Paley theorem holds for $\left\{E_{k}\right\}$ where $E_{k}=R_{k}-R_{k-1}$ and $\left\{R_{k}\right\}$ is an approximation to the identity, that is, for $1<p<\infty$,

$$
c\|f\|_{L_{B}^{p}} \leq\left\|\left\{\sum_{k \in Z}\left|E_{k}(f)\right|^{2}\right\}^{\frac{1}{2}}\right\|_{L_{B}^{p}} \leq C\|f\|_{L_{B}^{p}} .
$$

The main result in this note is the following:

Theorem 1.2. Suppose that $B$ is a Banach space. If the B-valued Littlewood-Paley theorem in (1.3) holds for some $1<p_{0}<\infty$ and $\left\{D_{k}\right\}$ where $D_{k}=S_{k}-S_{k-1}$ and $\left\{S_{k}\right\}$ is an approximation to the identity, then $B$ is isomorphic to a Hilbert space.

To prove Theorem 1.2, we will prove a result on general Banach spaces, Theorem 3.1 , in Section 3 and then Theorem 1.2 will be obtained by reducing to Kwapien's well-known characterization of Hilbert spaces.

\section{Proof of Theorem 1.1}

To show Theorem 1.1, we need a Calderón-type reproducing formula. More precisely, we first need the following definition.

Definition 2.1. Fix two exponents $0<\beta \leq 1$ and $\gamma>0$. A B-valued function $f$, where $B$ is a Banach space, is said to be a test function of type $(\beta, \gamma)$ centered at $x_{0} \in R^{n}$ with width $d>0$ if $f$ satisfies the following conditions:

$$
\begin{gathered}
|f(x)|_{B} \leq c \frac{d^{\gamma}}{\left(d+\left|x-x_{0}\right|\right)^{n+\gamma}} \\
\left|f(x)-f\left(x^{\prime}\right)\right|_{B} \leq c\left(\frac{\left|x-x^{\prime}\right|}{d+\left|x-x_{0}\right|}\right)^{\beta} \frac{d^{\gamma}}{\left(d+\left|x-x_{0}\right|\right)^{n+\gamma}}
\end{gathered}
$$


for $\left|x-x^{\prime}\right| \leq \frac{1}{2}\left(d+\left|x-x_{0}\right|\right)$,

$$
\int_{R^{n}} f(x) d x=0 .
$$

The collection of all test functions of type $(\beta, \gamma)$ centered at $x_{0}$ with width $d>0$ will be denoted by $M_{B}^{(\beta, \gamma)}\left(x_{0}, d\right)$. If $f \in M_{B}^{(\beta, \gamma)}\left(x_{0}, d\right)$, the norm of $f$ in $M_{B}^{(\beta, \gamma)}\left(x_{0}, d\right)$ is defined by

$$
\|f\|_{M_{B}^{(\beta, \gamma)}\left(x_{0}, d\right)}=\inf \{c \geq 0: \text { (i), (ii) and (iii) of Definition (2.1) hold }\} .
$$

We denote the class of all $f \in M_{B}^{(\beta, \gamma)}(0,1)$ by $M_{B}^{(\beta, \gamma)}$. It is easy to see that $M_{B}^{(\beta, \gamma)}$ is a Banach space under the norm $\|f\|_{M_{B}^{(\beta, \gamma)}}<\infty$. It is also easy to see that $M_{B}^{(\beta, \gamma)}=$ $M_{B}^{(\beta, \gamma)}\left(x_{0}, d\right)$, for $x_{0} \in R^{n}$ and $d>0$, with equivalent norms.

We now can state the following Calderón-type reproducing formula for $M_{B}^{(\beta, \gamma)}$

Theorem 2.1. Suppose that $\left\{S_{k}\right\}$ is an approximation to the identity defined in (1.3). Set $D_{k}=S_{k}-S_{k-1}$. Then there exists a family of operators $\left\{\widetilde{D}_{k}\right\}_{k \in Z}$ such that for all $f \in M_{B}^{(\beta, \gamma)}$,

$$
f=\sum_{k \in Z} \widetilde{D}_{k} D_{k}(f)
$$

where the series converges in the norm of $M_{B}^{\left(\beta^{\prime}, \gamma^{\prime}\right)}$ with $\beta^{\prime}<\beta$ and $\gamma^{\prime}<\gamma$. Moreover, $\widetilde{D}_{k}(x, y)$, the kernel of $\widetilde{D}_{k}$, satisfy the following estimates: for $\epsilon^{\prime}, 0<\epsilon^{\prime}<\epsilon$, where $\epsilon$ is the regularity exponent of $S_{k}$, there exists a constant $C>0$ such that

$$
\left|\widetilde{D}_{k}(x, y)\right| \leq C \frac{2^{-k \epsilon^{\prime}}}{\left(2^{-k}+|x-y|\right)^{n+\epsilon^{\prime}}}
$$

$$
\left|\widetilde{D}_{k}(x, y)-\widetilde{D}_{k}\left(x^{\prime}, y\right)\right| \leq C\left(\frac{\left|x-x^{\prime}\right|}{2^{-k}+|x-y|}\right)^{\epsilon^{\prime}} \frac{2^{-k \epsilon^{\prime}}}{\left(2^{-k}+|x-y|\right)^{n+\epsilon^{\prime}}}
$$

for $\left|x-x^{\prime}\right| \leq \frac{1}{2}\left(2^{-k}+|x-y|\right)$,

$$
\int \widetilde{D}_{k}(x, y) d y=\int \widetilde{D}_{k}(x, y) d x=0 \quad \text { for all } k \in Z .
$$

The proof of this theorem is similar to the scalar case and can be found in [1].

Since $\sum_{k \in Z} D_{k}(f)=f$ in the strong topology of $L_{B}^{2}\left(R^{n}\right)$, it is easy to see that $M_{B}^{(\beta, \gamma)}$ is dense in $L_{B}^{2}\left(R^{n}\right)$ for all $0<\beta \leq 1$ and $\gamma>0$. So to prove Theorem 1.1, we only need to show (1.4) for all $f \in M_{B}^{(\beta, \gamma)}$.

We are ready to prove Theorem 1.1. Suppose (1.3) holds and $E_{k}=R_{k}-R_{k-1}$ where $\left\{R_{k}\right\}$ is an approximation to the identity. By Theorem 2.1, for all $f \in M_{B}^{(\beta, \gamma)}$ with $0<\beta \leq 1$ and $\gamma>0$, we have

$$
E_{k}(f)=\sum_{j \in Z} E_{k} \widetilde{D}_{j} D_{j}(f)
$$

It is easy to check that $E_{k} \widetilde{D}_{j}(x, y)$, the kernel of $E_{k} \widetilde{D}_{j}$, satisfies the following estimates (see [1]):

$$
\left|E_{k} \widetilde{D}_{j}(x, y)\right| \leq c 2^{-|k-j| \epsilon^{\prime \prime}} \frac{2^{-(k \wedge j) \epsilon^{\prime \prime}}}{\left(2^{-(k \wedge j)}+|x-y|\right)^{n+\epsilon^{\prime \prime}}}
$$

where $0<\epsilon^{\prime \prime}<\epsilon^{\prime}<\epsilon$ and $a \wedge b$ denotes the minimum of $a$ and $b$. 
Hence

$$
\begin{aligned}
\left\|\left\{\sum_{k \in Z}\left|E_{k}(f)\right|^{2}\right\}^{\frac{1}{2}}\right\|_{p} & \leq c\left\|\left\{\sum_{k \in Z}\left[\sum_{j \in Z} 2^{-|k-j| \epsilon^{\prime \prime}} \mid M\left(D_{j}(f)\right)\right]^{2}\right\}^{\frac{1}{2}}\right\|_{p} \\
& \leq c\left\|\left\{\sum_{j \in Z}\left|M\left(D_{j}(f)\right)\right|^{2}\right\}^{\frac{1}{2}}\right\|_{p} \\
& \leq c\left\|\left\{\sum_{j \in Z}\left|D_{j}(f)\right|^{2}\right\}^{\frac{1}{2}}\right\|_{p}
\end{aligned}
$$

where $M$ is the Hardy-Littlewood maximal function; the last inequality follows from the Fefferman-Stein vector-valued maximal inequality.

The proof of the inverse inequality of (2.4) is the same, and, hence, this completes the proof of Theorem 1.1.

\section{Proof of Theorem 1.2}

To prove Theorem 1.2, we first observe that if (1.3) holds for some $1<p_{0}<\infty$, then (1.3) holds for all $1<p<\infty$. To see this, we define the operator $T$ on $L_{B}^{p_{0}}\left(R^{n}\right)$ by $T(f)=\left\{D_{k}(f)\right\}_{k \in Z}$. The fact that (1.3) holds for $p_{0}$ means that $T$ is a bounded operator from $L_{B}^{p_{0}}\left(R^{n}\right)$ to $L_{\mathcal{L}_{B}^{2}}^{p_{0}}\left(R^{n}\right)$ where

$$
L_{\mathcal{L}_{B}^{2}}^{p_{0}}\left(R^{n}\right)=\left\{\left(f_{k}(x)\right)_{k \in Z}:\left\|\left\{\sum_{k \in Z}\left|f_{k}(x)\right|_{B}^{2}\right\}^{\frac{1}{2}}\right\|_{p_{0}}<\infty\right\} .
$$

It is easy to check that $T$ is a vector-valued Calderón-Zygmund operator. Here we say that an operator $T$ is a vector-valued Calderón-Zygmund operator if $T$ is a continuous linear operator from $L_{B}^{p_{0}}\left(R^{n}\right)$ to $L_{\mathcal{L}_{B}^{2}}^{p_{0}}\left(R^{n}\right)$ for some $1<p_{0}<\infty$, with the kernel $K(x, y)$ mapping $R^{n} \times R^{n}$ to the space of all bounded operators from $B$ to $\mathcal{L}_{B}^{2}$ and satisfying the following conditions: for some $\epsilon>0$, there is a constant $C \geq 0$ such that

$$
\begin{gathered}
\|K(x, y)\| \leq C|x-y|^{-n} \quad \text { for all } x, y \in R^{n} \text { with } x \neq y, \\
\left\|K(x, y)-K\left(x, y^{\prime}\right)\right\| \leq C\left|y-y^{\prime}\right|^{\epsilon}|x-y|^{-(n+\epsilon)} \quad \text { for all } \quad\left|y-y^{\prime}\right| \leq \frac{1}{2}|x-y| \\
\left\|K(x, y)-K\left(x^{\prime}, y\right)\right\| \leq C\left|x-x^{\prime}\right|^{\epsilon}|x-y|^{-(n+\epsilon)} \quad \text { for all } \quad\left|x-x^{\prime}\right| \leq \frac{1}{2}|x-y| .
\end{gathered}
$$

By the Calderón-Zygmund real-variable theory, $T$ also is bounded from $\mathcal{L}_{B}^{p}\left(R^{n}\right)$ to $L_{L_{B}^{2}}^{p}\left(R^{n}\right)$ for all $1<p<\infty$.

Now the proof of Theorem 1.2 follows from a result on general Banach spaces. In the following statement, which is implicit in the literature, we will be using the well-known fact that lacunarity implies statistical independence.

Theorem 3.1. Let $B$ be any Banach space, $n \geq 1, a_{0} \in B, \ldots, a_{n} \in B$. Let $1<\lambda_{1}<$ $\cdots<\lambda_{n}<\lambda_{n+1}<\ldots, \lambda_{j}$ be integers for all $j$ and

$$
2 \pi\left\{\frac{\lambda_{1}}{\lambda_{2}}+\frac{\lambda_{1}+\lambda_{2}}{\lambda_{3}}+\cdots+\frac{\lambda_{1}+\lambda_{2}+\cdots+\lambda_{n}}{\lambda_{n+1}}+\cdots\right\} \leq \alpha<1 .
$$

Let $F_{n}\left(\theta_{1}, \ldots, \theta_{n}\right)=a_{0}+a_{1} e^{i \theta_{1}}+\cdots+a_{n} e^{i \theta_{n}}, 0 \leq \theta_{k} \leq 2 \pi$ for $1 \leq k \leq n$, and $f_{n}(t)=a_{0}+a_{1} e^{i \lambda_{1} t}+\cdots+a_{n} e^{i \lambda_{n} t}, 0 \leq t \leq 2 \pi$. Then,

$$
(1-\alpha)\left\|F_{n}\right\|_{2} \leq\left\|f_{n}\right\|_{2} \leq(1+\alpha)\left\|F_{n}\right\|_{2}
$$


where, and below, the $L^{2}$-norm will always be the normalized $L^{2}[0,2 \pi]^{n}$ norm or $L^{2}[0,2 \pi]$ with respect to all the variables.

To prove Theorem 3.1, set

$$
F_{n, k}\left(t, \theta_{k+1}, \ldots, \theta_{n}\right)=a_{0}+a_{1} e^{i \lambda_{1} t}+\cdots+a_{k} e^{i \lambda_{k} t}+a_{k+1} e^{i \theta_{k+1}}+\cdots+a_{n} e^{i \theta_{n}} .
$$

We will prove

$$
\left\|F_{n, k-1}\right\|_{2}-\epsilon_{k}\left\|F_{n}\right\|_{2} \leq\left\|F_{n, k}\right\|_{2} \leq\left\|F_{n, k-1}\right\|_{2}+\epsilon_{k}\left\|F_{n}\right\|_{2}
$$

where $1 \leq k \leq n$ and $\epsilon_{k}=2 \pi \frac{\lambda_{1}+\cdots+\lambda_{k-1}}{\lambda_{k}}$.

Observe first that once (3.7) is proved, we obviously obtain

$$
\left(1-\epsilon_{1}-\cdots-\epsilon_{n}\right)\left\|F_{n}\right\|_{2} \leq\left\|f_{n}\right\|_{2} \leq\left(1+\epsilon_{1}+\cdots+\epsilon_{n}\right)\left\|F_{n}\right\|_{2},
$$

which yields Theorem 3.1 .

The second observation is that the inequality in (3.7) for $1 \leq k<n$ follows from the inequality in (3.7) for $k=n$. Indeed, let us freeze $\theta_{k+1}, \ldots, \theta_{n}$ and write

$$
\tilde{a}_{0}=a_{0}+a_{k+1} e^{i \theta_{k+1}}+\cdots+a_{n} e^{i \theta_{n}} .
$$

We now apply (3.7) with $n$ being replaced by $k$, and then obtain

$$
\begin{gathered}
\left\|\widetilde{a}_{0}+a_{1} e^{i \lambda_{1} t}+\cdots+a_{k-1} e^{i \lambda_{k-1} t}+a_{k} e^{i \theta_{k}}\right\|_{L^{2}\left(d t d \theta_{k}\right)} \\
-\epsilon_{k}\left\|\widetilde{a}_{0}+a_{1} e^{i \theta_{1}}+\cdots+a_{k} e^{i \theta_{k}}\right\|_{L^{2}\left(d \theta_{1} \cdots d \theta_{k}\right)} \\
\leq\left\|\widetilde{a}_{0}+a_{1} e^{i \lambda_{1} t}+\cdots+a_{k} e^{i \lambda_{k} t}\right\|_{L^{2}(d t)} \\
\leq\left\|\widetilde{a}_{0}+a_{1} e^{i \lambda_{1} t}+\cdots+a_{k-1} e^{i \lambda_{k-1} t}+a_{k} e^{i \theta_{k}}\right\|_{L^{2}\left(d t d \theta_{k}\right)} \\
+\epsilon_{k}\left\|\widetilde{a}_{0}+a_{1} e^{i \theta_{1}}+\cdots+a_{k} e^{i \theta_{k}}\right\|_{L^{2}\left(d \theta_{1} \cdots d \theta_{k}\right) .}
\end{gathered}
$$

Writing $\phi$ for $\left(\theta_{k+1}, \ldots, \theta_{n}\right)$ and using symbolic notation, we have obtained

$$
A(\phi)-\epsilon_{k} B(\phi) \leq C(\phi) \leq A(\phi)+\epsilon_{k} B(\phi) .
$$

Now we take $L^{2}$ norms with respect to $\phi$ and obtain

$$
\begin{aligned}
\|A(\phi)\|_{L^{2}(d \phi)}-\epsilon_{k}\|B(\phi)\|_{L^{2}(d \phi)} & \leq\|C(\phi)\|_{L^{2}(d \phi)} \\
& \leq\|A(\phi)\|_{L^{2}(d \phi)}+\epsilon_{k}\|B(\phi)\|_{L^{2}(d \phi)} .
\end{aligned}
$$

Here we use the following observation: $f(x) \geq 0, g(x) \geq 0, h(x) \geq 0$, and $h(x) \geq$ $f(x)-g(x)$ imply $\|h\|_{2} \geq\|f\|_{2}-\|g\|_{2}$, since $h(x)+g(x) \geq f(x)$ obviously implies $\|h\|_{2}+\|g\|_{2} \geq\|h+g\|_{2} \geq\|f\|_{2}$.

Since $\|A(\phi)\|_{L^{2}(d \phi)}=\left\|F_{n, k-1}\right\|_{2},\|B(\phi)\|_{L^{2}(d \phi)}=\left\|F_{n}\right\|_{2}$, and $\|C(\phi)\|_{L^{2}(d \phi)}=$ $\left\|F_{n, k}\right\|_{2}$, the inequalities of (3.7) for $1 \leq k<n$ follows from (3.10).

It remains to prove the inequality of (3.7) with $k=n$. Note first that

$$
f_{n}\left(t+\frac{2 k \pi}{\lambda_{n}}\right)=f_{n-1}\left(t+\frac{2 k \pi}{\lambda_{n}}\right)+a_{n} e^{i \lambda_{n} t} .
$$

We now introduce

$$
f_{n}^{\#}(t, k, s)=f_{n-1}\left(t+\frac{2 k \pi}{\lambda_{n}}+\frac{2 \pi s}{\lambda_{n}}\right)+a_{n} e^{i \lambda_{n} t} \quad \text { if } 0 \leq s \leq 1 .
$$

Then

$$
\begin{aligned}
\left|f_{n}\left(t+\frac{2 k \pi}{\lambda_{n}}\right)-f_{n}^{\#}(t, k, s)\right|_{B} & \leq\left|a_{1}\right|_{B} \frac{2 \pi \lambda_{1}}{\lambda_{n}}+\cdots+\left|a_{n-1}\right|_{B} \frac{2 \pi \lambda_{n-1}}{\lambda_{n}} \\
& \leq \sup \left(\left|a_{1}\right|_{B}, \ldots,\left|a_{n-1}\right|_{B}\right) \epsilon_{n} .
\end{aligned}
$$


We obviously have

$$
\left|a_{k}\right|_{B} \leq\left\|F_{n}\right\|_{2} \quad \text { for } 1 \leq k \leq n
$$

since $a_{k}$ are the Fourier coefficients of $F_{n}$. Therefore,

$$
\left|f_{n}\left(t+\frac{2 k \pi}{\lambda_{n}}\right)-f_{n}^{\#}(t, k, s)\right|_{B} \leq \epsilon_{n}\left\|F_{n}\right\|_{2} .
$$

Taking the $L^{2}$ norm with respect to all the variables $t, k \in\left\{0,1, \ldots, \lambda_{n}-1\right\}$ and $s \in[0,1]$, we obtain

$$
\begin{aligned}
& \left|\left\{\frac{1}{\lambda_{n}} \sum_{k=0}^{\lambda_{n}-1}\left\|f_{n}\left(t+\frac{2 k \pi}{\lambda_{n}}\right)\right\|_{L^{2}(d t)}^{2}\right\}^{\frac{1}{2}}-\left\{\frac{1}{2 \pi} \int_{0}^{2 \pi} \int_{0}^{1} \frac{1}{\lambda_{n}} \sum_{k=0}^{\lambda_{n}-1}\left|f_{n}^{\#}(t, k, s)\right|^{2} d s d t\right\}^{\frac{1}{2}}\right| \\
& \leq \epsilon_{n}\left\|F_{n}\right\|_{2} .
\end{aligned}
$$

But

$$
\begin{aligned}
\int_{0}^{1} & \frac{1}{\lambda_{n}} \sum_{k=0}^{\lambda_{n}-1}\left|f_{n}^{\#}(t, k, s)\right|^{2} d s \\
& =\frac{1}{\lambda_{n}} \sum_{k=0}^{\lambda_{n}-1} \int_{0}^{1}\left|f_{n-1}\left(t+\frac{2 k \pi}{\lambda_{n}}+\frac{2 \pi s}{\lambda_{n}}\right)+a_{n} e^{i \lambda_{n} t}\right|^{2} d s \\
& =\frac{1}{2 \pi} \int_{0}^{2 \pi}\left|f_{n-1}(t+\theta)+a_{n} e^{i \lambda_{n} t}\right|^{2} d \theta \\
& =\frac{1}{2 \pi} \int_{0}^{2 \pi}\left|f_{n-1}(\theta)+a_{n} e^{i \lambda_{n} t}\right|^{2} d \theta .
\end{aligned}
$$

Then (3.12) yields

$$
\left|\left\|f_{n}(t)\right\|_{2}-\left\{\frac{1}{(2 \pi)^{2}} \int_{0}^{2 \pi} \int_{0}^{2 \pi}\left|f_{n-1}(\theta)+a_{n} e^{i \lambda_{n} t}\right|^{2} d \theta d t\right\}^{\frac{1}{2}}\right| \leq \epsilon_{n}\left\|F_{n}\right\|_{2},
$$

which is the required estimate.

We are ready to prove Theorem 1.2. Let $\psi, \phi \in S\left(R^{n}\right)$ with supp $\widehat{\psi} \subset\left\{\xi \in R^{n}\right.$ $\left.: \frac{1}{2} \leq|\xi| \leq 2\right\},|\widehat{\psi}(\xi)| \geq c>0$ if $\frac{3}{5} \leq|\xi| \leq \frac{5}{3}$, and supp $\widehat{\phi} \subset\left\{\xi \in R^{n}:|\xi| \leq\right.$ $1\}, \sup _{x \in[0,2 \pi]}|\phi(x)|^{2} \geq \delta>0$. Suppose that we accept the B-valued Littlewood-Paley theorem in (1.3) for some $1<p_{0}<\infty$ and $\left\{D_{k}\right\}$ where $D_{k}=S_{k}-S_{k-1}$ and $\left\{S_{k}\right\}$ is an approximation to the identity. By Theorem 1.1 and the first observation above, we may assume the following inequalities hold:

$$
c\|f\|_{L_{B}^{2}}^{2} \leq \sum_{k \in Z}\left\|\psi_{k} * f\right\|_{L_{B}^{2}}^{2} \leq C\|f\|_{L_{B}^{2}}^{2}
$$

where the constants $c$ and $C$ are independent of $f$.

Now consider the function $f(x)=f_{n}(x) \phi(x)=\left[a_{1} e^{i \lambda_{1} x}+\cdots+a_{n} e^{i \lambda_{n} x}\right] \phi(x)$ where $\lambda_{j}=3^{3^{j}}$ for $1 \leq j \leq n$. Then (3.15) implies

$$
\sum_{j=1}^{n}\left\|a_{j}\right\|_{B}^{2} \approx\left\|f_{n}\right\|_{L_{B}^{2}[0,2 \pi]}^{2}
$$

See [3] for the equivalence of the above norms.

We now apply Theorem 3.1 and obtain

$$
\left\|f_{n}\right\|_{L_{B}^{2}[0,2 \pi]}^{2} \approx\left\|a_{1} e^{i \theta_{1}}+\cdots+a_{n} e^{i \theta_{n}}\right\|_{L_{B}^{2}[0,2 \pi]^{n}}^{2} .
$$


By a result of [4] (Theorem 4.5 on page 313 ), we have

$$
\left\|a_{1} e^{i \theta_{1}}+\cdots+a_{n} e^{i \theta_{n}}\right\|_{L_{B}^{2}[0,2 \pi]^{n}}^{2} \approx \frac{1}{2^{n}} \sum_{\epsilon}\left\|\epsilon_{1} a_{1}+\cdots+\epsilon_{n} a_{n}\right\|_{B}^{2}
$$

where the series is extended over all sequences $\epsilon=\left(\epsilon_{1}, \ldots, \epsilon_{n}\right)$ with $\epsilon_{k}$ being independent Bernoulli random variables, that is, $\epsilon_{k}= \pm 1$ for $1 \leq k \leq n$.

This shows that for any $n \geq 1$ and $a_{1}, a_{2}, \ldots, a_{n} \in B$, there exist constants $c$ and $C$ such that

$$
c \sum_{j=1}^{n}\left\|a_{j}\right\|_{B}^{2} \leq \frac{1}{2^{n}} \sum_{\epsilon}\left\|\epsilon_{1} a_{1}+\cdots+\epsilon_{n} a_{n}\right\|_{B}^{2} \leq C \sum_{j=1}^{n}\left\|a_{j}\right\|_{B}^{2},
$$

which, together with a result of [2], implies that $B$ is isomorphic to a Hilbert space, and hence, Theorem 1.2 is proved.

\section{References}

1. Y.-S. Han, Calderón-type reproducing formula and the Tb theorem, Revista Matematica Iberoamericana 10 (1994), 51-91.

2. S. Kwapien, Isomorphic characterizations of inner product spaces by orthogonal series with vector valued coefficients, Studia Math. 44 (1972), 583-595.

3. G. Pisier, Les inegalites de Kahane-Khintchin d'apres C. Borell, Seminaire sur la geometrie des éspaces de Banach, Exposé No. VII (1977-1978), Ecole Polytechnique, Palaiseau.

4. N. N. Vakhania, V. I. Tarieladze, and S. A. Chobanyan, Probability Distributions on Banach Spaces, Mathematics and Its Applications (Soviet Series), D. Reidel Publishing Company, 1987.

Department of Mathematics, Auburn University, Auburn, Al 36849-5310, U.S.A.

CEREMade, Universite de Paris-Dauphine, Place De-Lattre-de-Tassigny, 75775 PaRis CEDEX 16 